\title{
Enrichment of redox-sensitive trace metals (U, V, Mo, As) associated with the late Hauterivian Faraoni oceanic anoxic event
}

\author{
Stéphane Bodin · Alexis Godet • Virginie Matera • \\ Philipp Steinmann · Jean Vermeulen · \\ Silvia Gardin · Thierry Adatte · Rodolfo Coccioni • \\ Karl B. Föllmi
}

Received: 6 July 2005 / Accepted: 6 March 2006/ Published online: 13 May 2006

(C) Springer-Verlag 2006

\begin{abstract}
The Faraoni Level is a short-lived oxygendeficient event that took place during the latest Hauterivian. In order to improve our understanding of the palaeoenvironmental conditions that occurred during this event, we have analysed the contents of several redox-sensitive trace elements $(\mathrm{U}, \mathrm{V}, \mathrm{Mo}, \mathrm{As}, \mathrm{Co}, \mathrm{Cd}$, $\mathrm{Cu}, \mathrm{Zn}, \mathrm{Ni}, \mathrm{Pb}, \mathrm{Cr}$ ) from bulk limestone samples of late Hauterivian-early Barremian age from three reference sections. U, V, Mo and As show consistent and significant enrichments during the Faraoni event whereas the other redox-sensitive trace elements analysed here are not systematically enriched. In order to explain this discrepant behaviour, we propose that the Faraoni Level was deposited during a period of anoxic conditions near the sediment-water interface. The distinctive peaks in U, V, Mo and As contents are traceable throughout the three studied sections and represent a good correlation tool which helps to identify the Faraoni Level and its equivalents in the western
\end{abstract}

S. Bodin $(\varangle) \cdot$ A. Godet $\cdot$ V. Matera $\cdot$ P. Steinmann

T. Adatte $\cdot$ K. B. Föllmi

Institut de Géologie, Université de Neuchâtel,

Rue Emile Argand 11, CP 158, 2009 Neuchatel, Switzerland

e-mail: stephane.bodin@unine.ch

J. Vermeulen

Grand Rue, 04330 Barrême, France

S. Gardin

CNRS-UMR 5143 "Paléodiversité et

Paléolenvironnement", case 104, Université Paris 6,

4 Place Jussieu, 75252 Paris Cedex 05, France

\section{R. Coccioni}

Istituto di Geologia e Centro di Geobiologia dell'Università, Campus Scientifico, Località Crocicchia,

61029 Urbino, Italy
Tethyan realm and outside of the Tethys. For example, a peak in $\mathrm{U}$ contents in upper Hauterivian sediments of the northwestern Pacific realm (ODP leg 185, site 1149) may well be an expression of the Faraoni event in this particular basin.

Keywords Hauterivian B Barremian ·

Chemostratigraphy $\cdot$ Western Tethys $\cdot$ Trace metals

\section{Introduction}

Since the first description by Cecca et al. (1994) of an organic-rich guide level-the so-called Faraoni Level-in upper Hauterivian (late Spathicrioceras angulicostatum ammonite zone) pelagic carbonates of the Umbria-Marche Basin (Italy), it has been shown that correlatable organic-rich levels occur throughout the western Tethyan realm. Equivalents of the Faraoni Level have been identified on the Trento Plateau in Italy (Cecca et al. 1996; Coccioni et al. 1998), in the Vocontian Trough in France (Baudin et al. 1999), and in the Swiss Préalpes (Busnardo et al. 2003). It is for this reason that Baudin et al. (2002) associated this level with a short-lived oxygen-deficient event in the western Tethyan realm. And following Baudin (2005), the Faraoni Level and its equivalent can be qualified as an anoxic event (compare this reference for a detailed biostratigraphic definition of this event). With the Valanginian event (Erba et al. 2004), the Faraoni event is one of the first palaeoceanographic events which led to major change in the carbon cycle during the early Cretaceous.

The environmental circumstances leading to this short-lived oxygen-deficient event in the late Hauterivian are still not clear. In contrast to the anoxic events 
in the Valanginian, the early Aptian, and near the Cenomanian/Turonian boundary (e.g. Schlanger and Jenkyns 1976; Schlanger et al. 1987; Lini et al. 1992; Jones and Jenkyns 2001; Sanfourche and Baudin 2001), the Faraoni Level is not accompanied by a major carbon isotope excursion toward more positive values, but rather by a small long-term increase culminating around the Faraoni event (Föllmi et al. 1994; Erba et al. 1999; Van de Schootbrugge et al. 2000; Company et al. 2005; Godet et al. 2006). Furthermore, no major volcanic episode has, of yet, been identified for the late Hauterivian. This means that the classical model postulated for the origin of oceanic anoxic events in general, i.e. increased volcanism leading to an increase in atmospheric $\mathrm{CO}_{2}$, climate warming, increased weathering and nutrient availability, higher productivity and consequently higher preservation rates of organic matter (e.g. Jenkyns 1999, 2003) cannot be applied in this case.

The Faraoni event corresponds in time to the onset of drowning episode D3 during the evolution of a carbonate platform system located along the northern Tethyan margin (Föllmi et al. 1994). But, in contrast to the Valanginian and the Aptian-Albian drowning episodes (D1, D4 and D5), the drowning episode D3-not being mirrored by a distinct positive excursion in carbon isotopes, is also in need of an alternative explanation (Van de Schootbrugge et al. 2000, 2003). It is therefore important to identify the palaeoenvironmental conditions leading to the Faraoni event, whilst also respecting the coeval drowning event on the northern Tethyan carbonate platform, which may have been linked to this anoxic event.

We investigated three (hemi-) pelagic key sections representing a north-south transect through the western Tethys and including micritic carbonates and marls of the Hauterivian-Barremian transition with regard to their redox-sensitive trace-element concentrations. The studied trace elements are uranium (U), vanadium $(\mathrm{V})$, molybdenum (Mo), arsenic (As), cobalt $(\mathrm{Co})$, cadmium $(\mathrm{Cd})$, cupper $(\mathrm{Cu})$, zinc $(\mathrm{Zn})$, nickel $(\mathrm{Ni})$, lead $(\mathrm{Pb})$ and chromium $(\mathrm{Cr})$. Their behaviour around the Faraoni Level is quite distinct and helps us to improve our understanding of redox conditions and changes therein in this basin during the Faraoni event.

In this paper, the term "Faraoni Level" and "Faraoni Level equivalent" refers to the interval composed of organic-rich sediments and the intervening carbonate beds that are associated with the Faraoni oceanic anoxic event. The term "Faraoni Level" is used when the lithological characteristics are the same as in the type area (e.g. Cecca et al. 1994), whereas the term "Faraoni Level equivalent" is used when the lithology is different from that of the type area (i.e. lacking a distinct enrichment in organic matter). The redox classification of sedimentary environments used here is adapted from Algeo and Maynard (2004) and references therein. Thus, oxic, suboxic and anoxic environments are characterized by $>2.0,2.0-0.2$ and $<0.2 \mathrm{ml} \mathrm{O}_{2} \mathrm{l}^{-1} \mathrm{H}_{2} \mathrm{O}$, respectively. Anoxia and euxinia are distinguished by the absence or presence of free $\mathrm{H}_{2} \mathrm{~S}$ in the water column, respectively.

\section{Studied sections and palaeogeographic setting}

The studied sections were chosen according to the following criteria: (1) presence of the Faraoni Level or its equivalent; (2) good age control based on biostratigraphy (ammonites, nannofossils); (3) presence of pelagic carbonates; and (4) representative of different areas of the western Tethys (Fig. 1).

The first studied section is the Fiume-Bosso section, located between Urbino and Gubbio, near Cagli (central Italy; see also Cecca et al. 1994). This section represents the type section for the Faraoni Level (Cecca et al. 1994). The lithology consists of pelagic limestone and chert, which locally includes thin organic-rich marl layers and laminae, characteristic of the Maiolica Formation. The Faraoni Level is composed by a succession of three laminated black-shale layers and intervening calcareous beds.

The Veveyse de Châtel-St Denis (VCD) is the second studied section. It is situated in the canton of Fribourg, along the Veveyse river, in western Switzerland (see Busnardo et al. 2003, for a detailed geographic description). Its lithology consists of a succession of alternating pelagic marls and marly limestones, which are rich in macrofossils. The equivalent of the Faraoni Level (identified by ammonite dating) is represented by the "couches à poissons" (an alternation of more or less organic-rich shale and marly limestone, rich in fish fossils) (Busnardo et al. 2003).

The third studied section is along the "route d'Angles," near Barrême in southeastern France, and is situated in the Vocontian Trough (see Busnardo 1965, for a detailed geographic description). Its lithology consists of a fossiliferous, hemi-pelagic succession of regularly alternating marlstone and limestone. Ammonite dating indicates that the Faraoni Level equivalent is situated around the bed AN 53. Black shale has not been distinguished within this interval, which is characterized by an alternation of limestone and laminated shale. In the sedimentary interval attributed to the $P$. mortilleti zone, no marls were sampled due to poor outcrop quality. 
Fig. 1 a Actual location of the three studied sections. b Paleogeographic position of the three studied sections at the Hauterivian-Barremian boundary. Palaeomap modified from Hay et al. (1999). Modified from Bodin et al. (2006)

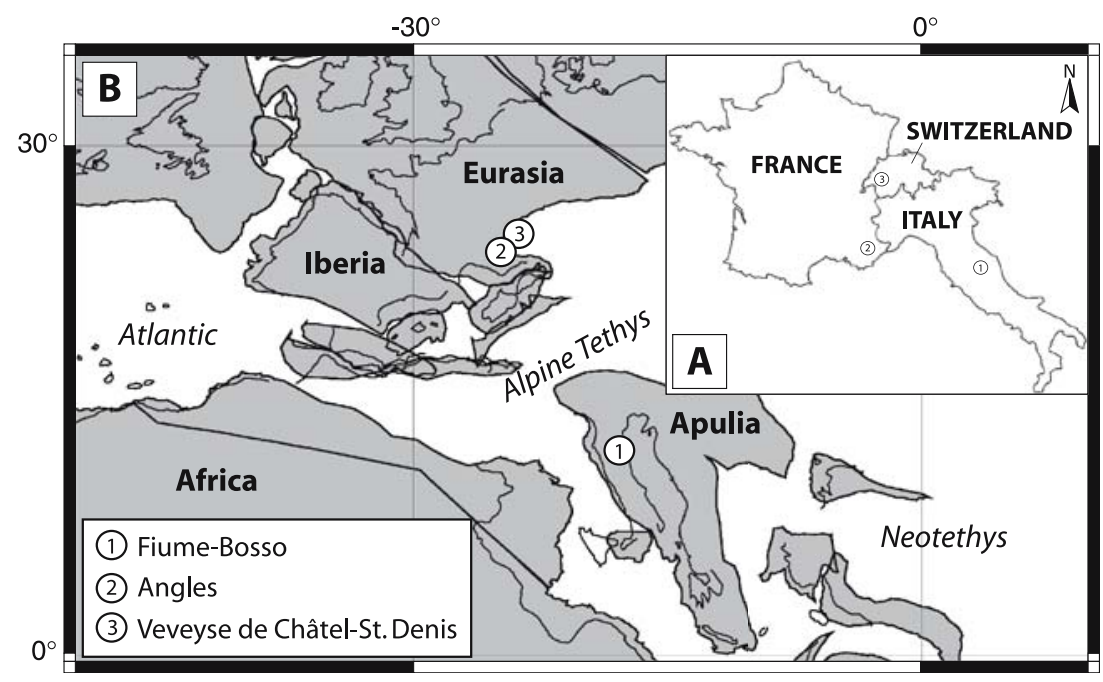

During the late Hauterivian, the Angles and the VCD sections were situated along the northern Tethyan margin. The VCD section is part of the Ultrahelvetic realm, which is considered as the deeper offshore prolongation of the Vocontian Trough to the northeast (Trümpy 1960). The Fiume-Bosso section, which is the deepest section, was situated in the southern part of the Tethys, in the Umbria-Marche Basin, remote from any continent.

\section{Methods}

ICP-MS analyses were performed on bulk rock samples from limestone and marlstone for all three sections. The limestone samples were sawed in order to eliminate altered parts and rock veins. Then, powders were obtained for both limestone and marls using a mechanic agate crusher. A portion of approximately $250 \mathrm{mg}$ was transferred into a digestion vessel and $10 \mathrm{ml}$ of concentrated nitric acid (suprapur, Merck, Darmstadt, Germany) were added. The sample was digested in a microwave oven (MSL-Ethos plus, Milestone) using the heating program recommended by the EPA 3051 method. After cooling, the resulting solution was filtered $(0.45 \mu \mathrm{m})$ and diluted to $100 \mathrm{ml}$ with ultrapure water. For the limestone, dissolution percentages determined after filtration were about 91\% of initial sample weight in the Angles section, $83 \%$ in the VCD section, and $98 \%$ in the Fiume-Bosso section. Moreover, no correlation was observed between the concentration of the different analysed samples and the dissolution percentage obtained during digestion procedure (e.g. $U$ in Fig. 2). This shows that the studied elements are present in the soluble authigenic phase and are not due to partial leaching of the detrital insoluble fraction.

In the marls, the dissolution percentages were close to $50 \%$. Mineralogical analyses indicate that clay and quartz grains constitute the insoluble part of the rock. No trace of carbonate is found, allowing us to postulate that the entire authigenic portion of the rock is dissolved with this method.

A second dilution (1/20) was then performed prior to analysis. To correct for matrix-induced ion signal variation and instrumental drift, rhodium was used as the internal standard. The element concentrations of the acid digests were determined by ICP-MS (PerkinElmer ELAN 6100, Perkin-Elmer Corp., Wellesley, MA, USA) using a semi-quantitative mode (Totalquant). Totalquant (TQ) is a simple, rapid and accurate panoramic method based on full mass-spectra scan methods and was successfully applied to the analysis of various biological and environmental samples (Jitaru et al. 2003) like, for instance, in sediments (Bayon et al. 1998). TQ enables spectral interpretation of a full mass spectrum by comparison with an internal response table which includes assignment of element intensities based on internal response factors and an interpretation of interferences based on implemented algorithms for numerical calculation of interference corrections. The response table is usually updated before analysis by determination of a blank and one standard solution. Two Certified Reference Materials (CRM) (NIST-1640 natural water, LKSD-1 lake sediment from Canadian Centre for Mineral and Energy Technology-CANMET-Canada) were measured to evaluate the accuracy which is achieved in panoramic analysis with the Elan 6100 . Here we present the results obtained for the four principal trace elements 


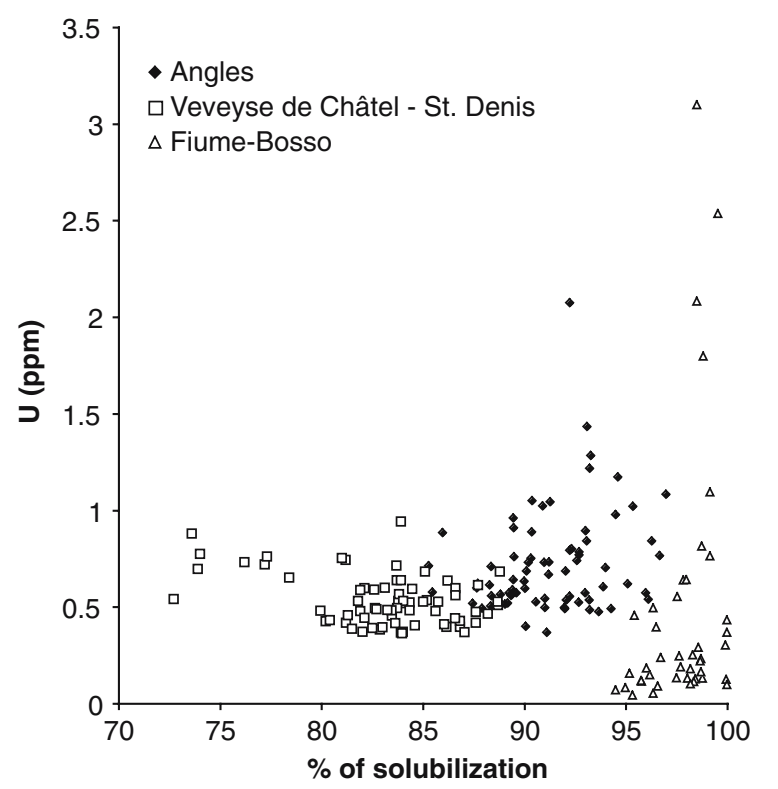

Fig. $2 U$ (ppm) versus percentage of dissolution in the three studied sections. No correlation is observed. See text for discussion

discussed in this study (V, Mo, As and $\mathrm{U})$. The mean recovery rates were determined for $\mathrm{V}, \mathrm{Mo}$ and As (no certified concentration values for $\mathrm{U}$ are available for the two CRM) and were, for the NIST-1640 CRM ( $N=11) 101 \%$ for $\mathrm{V}, 99 \%$ for Mo and $101 \%$ for As, and relative standard deviation (RSD) was better than $\pm 9 \%$ for $\mathrm{V}, \pm 6 \%$ for $\mathrm{Mo}$ and $\pm 7 \%$ for As; for the LKSD-1 CRM ( $N=12$ digestions) mean recovery rates were $90 \%$ for $\mathrm{V}, 89 \%$ for Mo and $110 \%$ for As (for this CRM, recovery rates are based on certified concentration values obtained by partial extraction using concentrated $\mathrm{HNO}_{3}$ and $\mathrm{HCl}$ whereas we used concentrated $\mathrm{HNO}_{3}$ digestion); analytical precision (RSD) was about $\pm 8 \%$ for $\mathrm{V}, \pm 9 \%$ for Mo and $\pm 12 \%$ for As.

Furthermore, multiple digestions $(N=10)$ of a sample corresponding to the Faraoni Level of the Angles section (bed AN 53-2) were analysed. The RSD of these analyses were about $\pm 15 \%$ for $\mathrm{V}, \pm 12 \%$ for Mo, $\pm 12 \%$ for As and $\pm 3 \%$ for U. A detection limit lower than $1 \mu \mathrm{g} / \mathrm{kg}$ was observed for the four trace elements studied.

The content and the type of organic matter were determined using Rock-Eval pyrolysis (Rock-Eval 6; Behar et al. 2001). The parameters TOC (total organic carbon in weight \%) was obtained using the standard temperature cycle. Two standards (an in-house standard and the "IFP 160000" standard from the Institut Français du Pétrole, Paris, France) were passed at the beginning and at the end of a batch of approximately 15 samples.

\section{Results}

Redox-sensitive trace elements

The data for $\mathrm{U}, \mathrm{V}, \mathrm{Mo}, \mathrm{As}, \mathrm{Co}, \mathrm{Cd}, \mathrm{Cu}, \mathrm{Zn}, \mathrm{Ni}, \mathrm{Pb}$ and $\mathrm{Cr}$ are shown in Tables 1 and 2 for limestone and marl for the three sections.

\section{The Fiume-Bosso section}

In limestone samples, the data obtained for $\mathrm{U}, \mathrm{V}, \mathrm{Mo}$ and As contents show comparable variations, with a consistently low background level, contrasted by a maximum in concentrations in and close to the Faraoni Level (Fig. 3; Table 1). The average values for the background level correspond to approximately $0.2,0.9$, 0.03 and $0.3 \mathrm{ppm}$ and the maximum values to approximately 3.1, 11.6, 0.5 and $3.0 \mathrm{ppm}$, for the U, V, Mo and As contents, respectively.

The data obtained in the marlstones show equally a clear enrichment of U, V, Mo and As around the Faraoni Level (Table 2). The maximum values reach $15.4 \mathrm{ppm}$ for $\mathrm{U}, 345.9 \mathrm{ppm}$ for $\mathrm{V}, 61.8 \mathrm{ppm}$ for Mo and $177.4 \mathrm{ppm}$ for As, whereas background level are equal to approximately $2.9,54.1,2.3$ and $13.2 \mathrm{ppm}$, respectively.

Despite a small negative shift in the upper part of the section, the global trend of Co in limestone samples seems to be more or less constant (Table 1), fluctuating around $1.8 \mathrm{ppm}$. The Faraoni Level is marked by a short negative shift toward $0.9 \mathrm{ppm}$. In the shale, the Co curve shows a small positive shift within the Faraoni Level. A maximum of $106.6 \mathrm{ppm}$ is present just above the Faraoni Level (Table 2) whereas the background level values correspond to approximately $50 \mathrm{ppm}$.

For the other elements, only $\mathrm{Cd}$ and $\mathrm{Zn}$ show a small positive shift in limestone levels within the Faraoni Level. In the upper part of the section, a positive shift (up to $66.1 \mathrm{ppm}$ ) of $\mathrm{Zn}$ is remarked. In the Faraoni shale samples, a positive shift of $\mathrm{Cd}, \mathrm{Cu}, \mathrm{Zn}$, $\mathrm{Ni}$ is present, with very high maximum values (10.5, 273.7, 1068.2 and $533.5 \mathrm{ppm}$, respectively). On the other hand, it appears that $\mathrm{Pb}$ and $\mathrm{Cr}$ record a negative shift.

\section{The VCD section}

In this section, the data obtained in limestone samples for $\mathrm{U}, \mathrm{V}, \mathrm{Mo}$ and As contents show a behaviour similar to that of the Fiume-Bosso section, with the difference that peaks are less pronounced in comparison to the 
Table 1 Redox-sensitive trace elements analyses in limestones for the three studied sections (expressed in ppm). The dark grey band indicates the Faraoni Level or its equivalent. No value is indicated when below the limit of detection

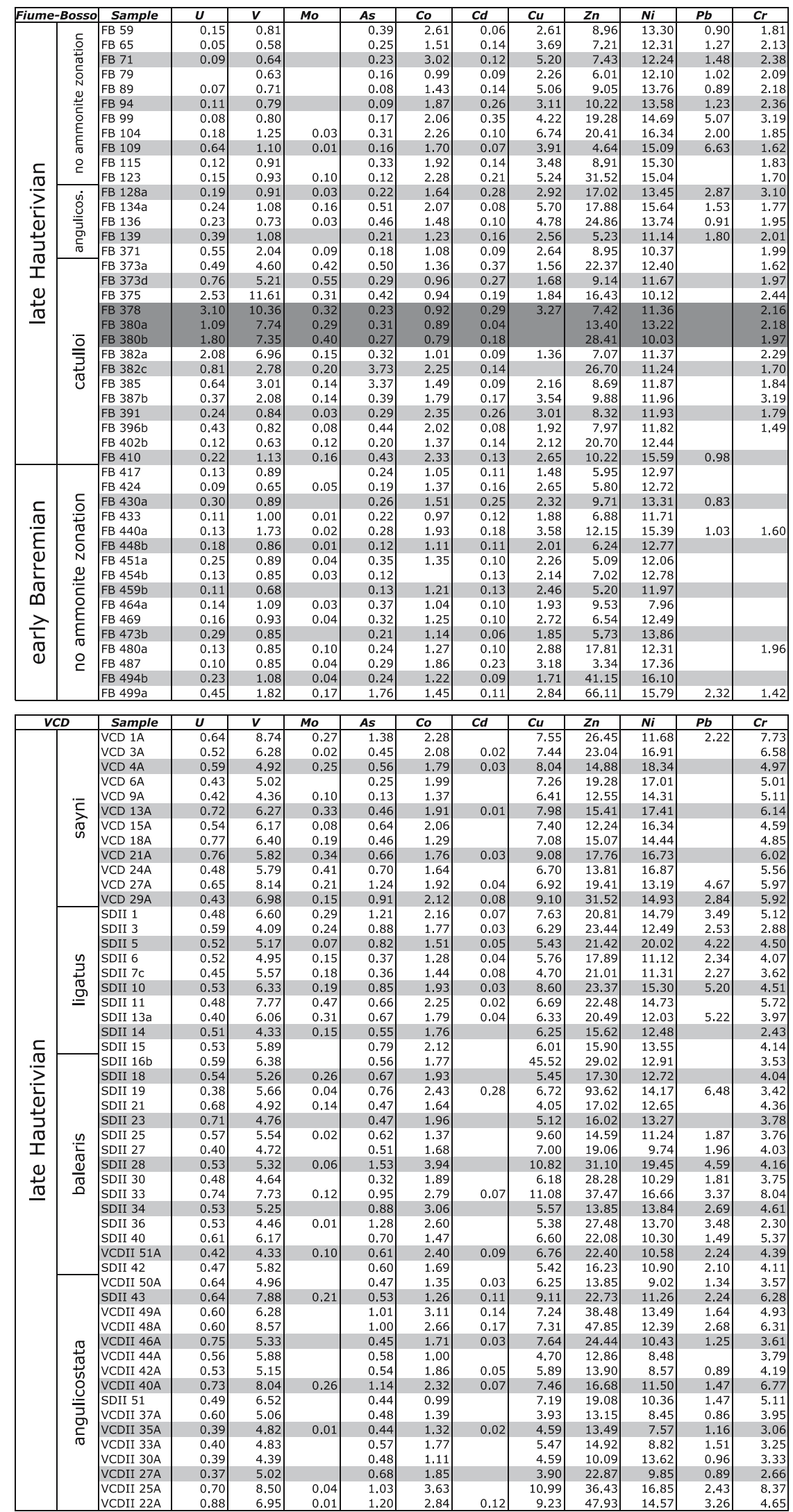


Table 1 continued

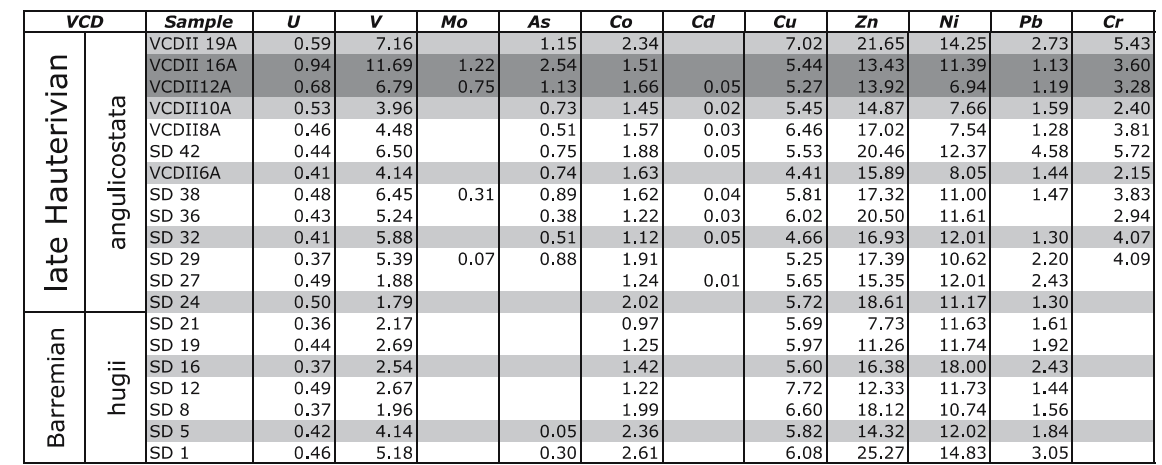

\begin{tabular}{|c|c|c|c|c|c|c|c|c|c|c|c|c|c|}
\hline \multicolumn{2}{|c|}{ Angles } & Sample & $U$ & $\boldsymbol{v}$ & Mo & As & Co & $C d$ & $\mathrm{Cu}$ & $Z n$ & $\mathrm{Ni}$ & $\mathbf{P b}$ & $\mathrm{Cr}$ \\
\hline \multirow{31}{*}{ 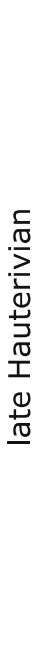 } & \multirow{17}{*}{ 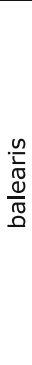 } & AN 2 & 0.57 & 7.78 & 0.60 & 0.82 & \begin{tabular}{l|l|}
1.04 \\
\end{tabular} & 0.02 & 5.54 & 16.12 & 13.14 & 1.52 & 6.69 \\
\hline & & AN 4 & 0.50 & 4.88 & 0.26 & 0.65 & 1.36 & 0.02 & 4.47 & 17.42 & 12.63 & 2.00 & 3.87 \\
\hline & & AN 6 & 0.60 & 5.04 & 0.20 & 0.77 & 1.49 & 0.03 & 4.68 & 21.93 & 13.41 & 2.35 & 3.68 \\
\hline & & AN 8 & 0.71 & 5.03 & 0.33 & 0.44 & 1.01 & 0.06 & 5.91 & 35.59 & 12.25 & 1.25 & 4.79 \\
\hline & & AN 10 & 0.88 & 4.87 & 0.14 & 0.77 & 1.79 & 0.02 & 5.79 & 15.46 & 13.57 & 1.54 & 4.44 \\
\hline & & AN 13 & 0.64 & 3.64 & 0.11 & 0.29 & 0.97 & 0.01 & 3.72 & 13.18 & 11.18 & & 3.29 \\
\hline & & AN 15.1 & 0.57 & 2.62 & 0.19 & 0.39 & 1.23 & 0.03 & 3.58 & 11.36 & 11.29 & 1.05 & 2.75 \\
\hline & & AN 16 & 0.52 & 2.75 & 0.14 & 0.36 & 1.28 & & 3.64 & 7.65 & 11.24 & 1.28 & 2.33 \\
\hline & & AN 18 & 0.55 & 3.89 & 0.40 & 0.45 & 1.32 & 0.05 & 4.88 & 30.37 & 11.16 & & 3.70 \\
\hline & & AN 20 & 0.49 & 3.43 & 0.45 & 0.25 & 0.80 & 0.04 & 3.11 & 17.93 & 8.95 & 0.91 & 3.41 \\
\hline & & AN 23 & 0.91 & 4.13 & 0.16 & 0.41 & 1.36 & 0.07 & 6.66 & 17.79 & 12.45 & 1.48 & 3.61 \\
\hline & & AN 26 & 0.76 & 2.92 & 0.18 & 0.30 & 0.83 & & 4.32 & 14.90 & 9.81 & 0.98 & 3.79 \\
\hline & & AN 27.2 & 0.62 & 4.64 & 0.25 & 0.66 & 1.15 & 0.11 & 6.85 & 26.72 & 11.30 & 1.91 & 4.43 \\
\hline & & AN 29 & 0.55 & 2.70 & 0.44 & 0.73 & 1.11 & 0.03 & 3.35 & 13.45 & 10.79 & 1.55 & 2.63 \\
\hline & & AN 31 & 0.67 & 3.04 & 0.17 & 0.61 & 1.17 & 0.02 & 4.61 & 20.26 & 11.28 & 1.24 & 3.18 \\
\hline & & AN 33 & 0.47 & 2.71 & 0.11 & 0.40 & 1.06 & & 3.42 & 6.85 & 10.55 & 1.16 & 3.11 \\
\hline & & AN 35 & 1.02 & 4.02 & 0.13 & 0.50 & 1.05 & 0.09 & 5.49 & 16.32 & 11.66 & 1.62 & 2.99 \\
\hline & \multirow{9}{*}{ 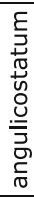 } & AN 38 & 0.48 & 2.45 & 1.02 & 1.49 & 5.15 & 0.39 & 5.92 & 75.44 & 24.32 & 6.24 & 2.39 \\
\hline & & AN 40 & 0.63 & 4.15 & 0.57 & 0.61 & 0.98 & 0.20 & 4.40 & 23.53 & 10.43 & 1.99 & 3.30 \\
\hline & & AN 43 & 0.73 & 3.97 & 0.24 & 0.57 & 1.25 & 0.09 & 5.67 & 18.99 & 11.65 & 1.93 & 3.81 \\
\hline & & AN 46 & 0.49 & 2.85 & 0.06 & 0.35 & 1.08 & 0.04 & 3.60 & 15.65 & 11.06 & 0.96 & 2.90 \\
\hline & & AN 49.1 & 0.73 & 4.18 & 0.10 & 0.43 & 1.13 & 0.11 & 3.93 & 48.61 & 10.54 & 1.13 & 3.00 \\
\hline & & AN 50 & 0.49 & 3.45 & 0.36 & 0.35 & 0.77 & 0.06 & 3.22 & 14.54 & 9.72 & 1.21 & 2.65 \\
\hline & & AN 51 & 1.05 & 4.62 & 0.59 & 0.74 & 1.16 & 0.08 & 5.34 & 26.19 & 12.53 & 1.58 & 3.48 \\
\hline & & AN 52 & 1.43 & 8.93 & 4.87 & 4.80 & 2.36 & 2.12 & 6.41 & 420.80 & 26.63 & 5.73 & 2.14 \\
\hline & & AN 53.2 & 1.28 & 7.43 & 4.61 & 3.45 & 1.42 & 0.05 & 3.83 & 13.13 & 23.69 & 4.81 & 1.99 \\
\hline & \multirow{5}{*}{ 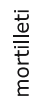 } & AN 55 & 2.07 & 4.75 & 0.14 & 0.20 & 0.71 & 0.03 & 3.16 & 25.79 & 8.58 & 1.02 & 2.35 \\
\hline & & AN 62.1 & 0.78 & 4.79 & 0.44 & 0.64 & 1.03 & & 3.52 & 17.13 & 10.21 & 1.08 & 2.45 \\
\hline & & AN 65.1 & 0.98 & 4.38 & 0.16 & 0.54 & 1.56 & & 3.93 & 12.59 & 11.89 & 2.06 & 1.87 \\
\hline & & AN 68 & 0.79 & 4.61 & 0.20 & 0.46 & 0.98 & 0.06 & 4.69 & 24.61 & 10.37 & 1.03 & 3.13 \\
\hline & & AN 71 & 0.89 & 4.22 & 0.06 & 0.47 & 0.99 & & 3.70 & 15.91 & 10.74 & 1.22 & 2.40 \\
\hline \multirow{41}{*}{ 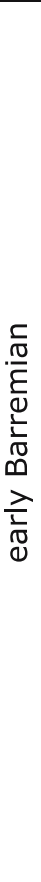 } & & AN 72 & 1.17 & 4.61 & & 0.31 & 1.05 & 0.12 & 4.14 & 23.89 & 11.19 & 1.91 & 2.22 \\
\hline & & AN 75 & 0.53 & 3.57 & 0.05 & 0.38 & 1.03 & 0.02 & 3.44 & 16.25 & 10.10 & 1.15 & 2.76 \\
\hline & & AN 78 & 0.54 & 5.00 & 0.17 & 0.62 & 2.17 & 0.04 & 4.18 & 29.54 & 11.93 & 1.57 & 3.40 \\
\hline & & AN 81 & 0.73 & 5.30 & 0.04 & 0.50 & 1.30 & 0.08 & 4.84 & 33.86 & 10.64 & 1.51 & 2.83 \\
\hline & $\frac{5}{\sigma}$ & AN 84 & 0.52 & 4.88 & 0.18 & 0.42 & 1.28 & 0.04 & 3.50 & 26.82 & 11.05 & 1.35 & 2.44 \\
\hline & $\stackrel{\underline{=}}{=}$ & AN 87 & 0.59 & 7.34 & 0.61 & 0.88 & 1.57 & 0.04 & 5.15 & 17.40 & 12.42 & 2.05 & 5.31 \\
\hline & $\underline{x}$ & AN 89 & 0.56 & 5.17 & 0.21 & 0.61 & 1.25 & 0.03 & 3.89 & 14.02 & 9.25 & 1.26 & 3.48 \\
\hline & & AN 90 & 0.56 & 4.98 & 0.29 & 0.86 & 1.79 & & 4.52 & 18.55 & 13.17 & 2.06 & 3.59 \\
\hline & & AN 92 & 0.40 & 4.08 & 0.20 & 0.58 & 1.34 & 0.04 & 4.55 & 12.24 & 11.60 & 2.37 & 3.18 \\
\hline & & AN 93 & 0.37 & 3.59 & 0.03 & 0.46 & 1.25 & 0.02 & 3.62 & 16.10 & 11.02 & 1.36 & 3.52 \\
\hline & & AN 95 & 0.51 & 3.95 & 0.21 & 0.87 & 1.47 & 0.06 & 5.80 & 17.18 & 10.65 & 1.74 & 3.31 \\
\hline & & AN 97 & 0.49 & 3.13 & 0.07 & 0.42 & 1.11 & 0.06 & 3.85 & 15.57 & 11.56 & 1.26 & 2.64 \\
\hline & $\overline{5}$ & AN 98 & 0.53 & 3.49 & 0.21 & 0.75 & 1.50 & 0.11 & 11.51 & 29.09 & 10.91 & 1.40 & 2.71 \\
\hline & $\frac{\omega}{x}$ & AN 101 & 0.71 & 4.92 & 0.23 & 0.92 & 1.33 & 0.04 & 6.11 & 23.13 & 12.82 & 2.21 & 3.65 \\
\hline & U & AN 103 & 0.57 & 6.88 & 0.65 & 1.02 & 2.01 & 0.06 & 6.03 & 30.35 & 12.74 & 2.42 & 4.53 \\
\hline & C & AN 104 & 0.52 & 5.15 & 0.37 & 1.10 & 1.30 & 0.06 & 6.05 & 24.55 & 11.33 & 2.22 & 4.06 \\
\hline & & AN 105 & 0.52 & 4.47 & 0.63 & 1.22 & 1.49 & 0.05 & 6.94 & 33.44 & 11.80 & 2.28 & 3.79 \\
\hline & & AN 108 & 0.89 & 5.81 & 0.71 & 1.08 & 1.49 & 0.08 & 6.21 & 37.61 & 11.22 & 2.20 & 3.37 \\
\hline & & AN 109.4 & 0.49 & 4.14 & 0.69 & 1.14 & 1.72 & 0.02 & 5.75 & 29.79 & 11.49 & 2.08 & 3.04 \\
\hline & $\underline{\underline{0}}$ & AN $110.1 \mathrm{~b}$ & 0.68 & 5.48 & 1.11 & 1.36 & 1.73 & 0.04 & 5.34 & 52.45 & 13.14 & 2.66 & 3.08 \\
\hline & $\overline{\bar{\alpha}}$ & AN $110.3 \mathrm{C}$ & 0.62 & 3.99 & 0.33 & 0.67 & 1.38 & 0.08 & 4.04 & 47.02 & 10.42 & 0.91 & 2.64 \\
\hline & $\frac{5}{0}$ & AN 110.4 & 1.04 & 5.03 & 0.47 & 1.07 & 1.09 & 0.07 & 5.29 & 21.30 & 11.21 & 1.77 & 2.88 \\
\hline & $\frac{0}{5}$ & AN 111.2 & 0.80 & 5.36 & 0.12 & 0.87 & 0.88 & 0.05 & 4.58 & 20.12 & 8.83 & 1.50 & 2.58 \\
\hline & $\vec{a}$ & AN 111.5 & 1.22 & 7.64 & 0.72 & 1.12 & 1.20 & 0.09 & 5.10 & 65.66 & 10.84 & 1.86 & 3.26 \\
\hline & & AN 112.2 & 0.74 & 6.66 & 0.17 & 0.55 & 1.33 & 0.07 & 4.41 & 23.15 & 10.43 & 1.24 & 2.52 \\
\hline & & AN 112.7 & 0.59 & 5.55 & 0.42 & 0.63 & 1.04 & 0.08 & 4.12 & 150.20 & 10.55 & 1.83 & 2.41 \\
\hline & $\underline{E}$ & AN 114.2 & 0.68 & 4.61 & 0.41 & 0.88 & 0.93 & 0.04 & 4.68 & 24.82 & 10.13 & 1.43 & 1.80 \\
\hline & $\frac{\overline{5}}{2}$ & AN 115 & 0.77 & 6.43 & 0.62 & 1.21 & 1.05 & 0.03 & 5.45 & 24.46 & 13.81 & 9.36 & 2.57 \\
\hline & $y^{2}$ & AN 117 & 0.60 & 4.16 & 0.30 & 0.74 & 1.27 & 0.05 & 5.44 & 24.94 & 10.40 & 1.36 & 1.87 \\
\hline & 产 & AN 119 & 0.57 & 3.10 & 0.23 & 0.34 & 1.00 & 1.25 & 4.83 & 80.29 & 10.27 & & 1.55 \\
\hline & E⿱ & AN 121 & 0.54 & 3.38 & 0.12 & 0.32 & 1.34 & 0.06 & 3.29 & 18.69 & 10.94 & 0.99 & 1.86 \\
\hline & U & AN 123 & 0.57 & 4.26 & 0.27 & 0.53 & 1.38 & 0.08 & 4.57 & 30.62 & 11.74 & 1.10 & 2.53 \\
\hline & & AN 125 & 0.96 & 6.99 & 0.73 & 1.06 & 1.95 & 0.05 & 7.09 & 41.84 & 14.53 & 1.94 & 3.81 \\
\hline & & AN 127 & 1.02 & 4.27 & 0.32 & 0.36 & 0.76 & 0.03 & 5.15 & 18.56 & 8.52 & & 1.98 \\
\hline & & AN 128 & 0.76 & 3.24 & 0.34 & 0.42 & 0.84 & 0.07 & 4.39 & 14.86 & 9.74 & & 1.69 \\
\hline & $\bar{n}$ & AN 129 & 0.75 & 4.25 & 1.70 & 2.58 & 1.76 & 0.09 & 6.48 & 23.92 & 12.89 & 1.88 & 2.28 \\
\hline & $\frac{1}{2}$ & AN 131 & 0.84 & 8.34 & 0.73 & 0.95 & 1.25 & 0.03 & 7.52 & 21.95 & 13.50 & 1.47 & 1.90 \\
\hline & $\frac{\pi}{0}$ & AN 133 & 0.84 & 3.10 & 0.39 & 1.08 & 1.00 & 0.10 & 6.30 & 21.42 & 12.97 & 1.45 & 2.01 \\
\hline & & AN 136.2 & 0.61 & 4.88 & 0.47 & 1.94 & 2.27 & 0.07 & 8.46 & 29.82 & 14.45 & 3.71 & 2.89 \\
\hline & & AN 138 & 0.70 & 3.41 & 0.57 & 1.10 & 0.97 & 0.21 & 6.72 & 44.43 & 11.66 & 1.36 & 1.17 \\
\hline & & AN 139d & 1.08 & 3.54 & 0.16 & 0.63 & 0.94 & 0.17 & 4.86 & 26.74 & 11.87 & 1.95 & 3.74 \\
\hline
\end{tabular}

background level (Fig. 4; Table 1). We also note that the background levels are generally higher in the lower part of the section, below the "couches à poissons"
(Faraoni Level equivalent), with the exception of Mo, which shows an interval of increased values only in the first $40 \mathrm{~m}$ of the measured section. Interestingly, $\mathrm{U}$ and 
Table 2 Redox-sensitive trace-element analyses in marlstones for the three studied sections (expressed in ppm). The dark grey band indicates the Faraoni Level or its equivalent. No value is indicated when below the limit of detection

\begin{tabular}{|c|c|c|c|c|c|c|c|c|c|c|c|c|c|}
\hline \multicolumn{2}{|c|}{ Fiume-Bo } & Sample & $\mathbf{U}$ & $\mathbf{v}$ & Mo & As & Co & Cd & $\mathrm{Cu}$ & $Z \mathbf{n}$ & $\mathbf{N i}$ & $\mathbf{P b}$ & $\mathrm{Cr}$ \\
\hline \multirow{8}{*}{ 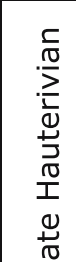 } & \multirow{3}{*}{$\begin{array}{l}\vec{\sigma} \\
\frac{\Gamma}{\pi}\end{array}$} & FB 133 & 2.01 & 69.51 & 2.96 & 15.55 & 39.00 & 1.62 & 93.32 & 287.33 & 136.27 & 39.08 & 38.66 \\
\hline & & Fb 138 & 3.97 & 27.74 & 1.00 & 19.77 & 41.14 & 1.20 & 139.33 & 235.05 & 99.30 & 26.68 & 33.83 \\
\hline & & FB 372 & 9.47 & 84.72 & 1.90 & 14.29 & 58.25 & 3.38 & 214.15 & 528.47 & 223.99 & 50.01 & 53.0 \\
\hline & \multirow{6}{*}{$\frac{\overline{0}}{\bar{\Xi}}$} & FB 374 & 12.83 & 345.94 & 61.83 & 177.42 & 84.54 & 10.48 & 270.70 & 1068.19 & 533.54 & 49.22 & 66.55 \\
\hline & & FB 377 & 6.29 & 68.15 & 15.97 & 8.13 & 9.42 & 4.32 & 23.68 & 220.95 & 99.15 & 4.49 & 8.79 \\
\hline & & FB 379 & 15.40 & 306.71 & 19.28 & 28.51 & 67.00 & 4.23 & 273.71 & 701.05 & 428.86 & 33.57 & 71.43 \\
\hline & & FB 381b & 9.92 & 151.09 & 28.26 & 33.44 & 29.64 & 5.06 & 61.67 & 313.80 & 268.18 & 14.52 & 22.21 \\
\hline & & FB 384 & 12.04 & 169.53 & 5.48 & 40.58 & 106.59 & 3.83 & 211.07 & 612.52 & 277.61 & 51.44 & $66.4 \varepsilon$ \\
\hline & & FB 397 & 2.85 & 20.43 & 0.30 & 8.25 & 67.51 & 0.90 & 164.07 & 248.47 & 143.25 & 34.58 & 33.60 \\
\hline
\end{tabular}

\begin{tabular}{|c|c|c|c|c|c|c|c|c|c|c|c|c|c|}
\hline \multicolumn{2}{|c|}{ VCD } & Sample & $\mathbf{U}$ & $\mathbf{V}$ & Mo & As & Co & Cd & $\mathrm{Cu}$ & Zn & $\mathbf{N i}$ & $\mathbf{P b}$ & $\mathrm{Cr}$ \\
\hline \multirow{10}{*}{ } & \multirow{15}{*}{ 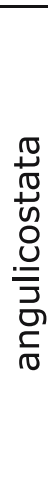 } & VCDII $10 \mathrm{~b}$ & 1.38 & 12.59 & 0.45 & 1.89 & 4.14 & 0.13 & 14.62 & 50.26 & 19.43 & 2.25 & 12.93 \\
\hline & & VCDII $11 b$ & 1.29 & 8.27 & 0.29 & 1.87 & 3.84 & 0.21 & 13.77 & 49.12 & 21.39 & 3.79 & 8.43 \\
\hline & & VCDII 14b & 0.82 & 5.47 & 0.57 & 1.95 & 3.00 & 0.05 & 10.19 & 20.06 & 19.01 & 1.90 & 7.22 \\
\hline & & VCDII $17 b$ & 3.27 & 15.21 & 1.59 & 6.74 & 3.46 & 0.60 & 15.77 & 53.27 & 23.53 & 3.95 & 8.34 \\
\hline & & VCDII $18 b$ & 1.26 & 11.67 & 0.12 & 2.37 & 4.93 & 0.05 & 16.14 & 51.92 & 27.56 & 4.30 & 13.63 \\
\hline & & VCDII 20b & 1.45 & 11.60 & & 3.44 & 7.42 & 0.20 & 18.30 & 86.81 & 37.34 & 9.23 & 11.95 \\
\hline & & VCDII 25b & 1.08 & 8.15 & & 1.85 & 5.13 & 0.43 & 19.39 & 112.65 & 28.29 & 4.37 & 9.33 \\
\hline & & VCDII 26b & 1.05 & 8.56 & & 2.68 & 4.41 & 0.13 & 15.84 & 56.10 & 23.71 & 5.68 & 9.18 \\
\hline & & VCDII 27b & 1.31 & 11.47 & 0.21 & 1.94 & 4.38 & 0.07 & 20.00 & 49.23 & 28.48 & 3.75 & 13.25 \\
\hline & & VCDII $28 b$ & 1.06 & 13.37 & 0.15 & 2.16 & 5.01 & 0.16 & 16.50 & 61.06 & 21.64 & 4.25 & 12.78 \\
\hline \multirow{5}{*}{$\underset{\underline{t}}{\underline{0}}$} & & VCDII 29b & 0.91 & 7.75 & 0.06 & 1.47 & 3.00 & 0.13 & 14.43 & 36.53 & 20.36 & 3.41 & 7.89 \\
\hline & & VCDII $30 \mathrm{~b}$ & 1.03 & 6.84 & 0.03 & 1.44 & 4.01 & 0.07 & 17.12 & 41.84 & 22.74 & 3.64 & 8.05 \\
\hline & & VCDII 31b & 1.17 & 6.99 & 0.19 & 2.09 & 5.13 & 0.06 & 15.28 & 36.51 & 26.18 & 5.04 & 8.26 \\
\hline & & VCDII 32b & 1.24 & 9.64 & 0.03 & 1.52 & 4.43 & 0.17 & 19.81 & 47.95 & 28.04 & 3.25 & 13.09 \\
\hline & & VCDII 33b & 1.50 & 11.92 & 0.17 & 2.30 & 4.63 & 0.10 & 24.18 & 65.32 & 34.76 & 6.90 & 13.59 \\
\hline
\end{tabular}

\begin{tabular}{|c|c|c|c|c|c|c|c|c|c|c|c|c|c|}
\hline \multicolumn{2}{|c|}{ Angles } & Sample & $\mathbf{U}$ & $\mathbf{v}$ & Mo & As & Co & Cd & $\mathrm{Cu}$ & $\mathrm{Zn}$ & $\mathbf{N i}$ & $\mathbf{P b}$ & $\mathrm{Cr}$ \\
\hline \multirow{3}{*}{$\frac{\check{\tau}}{\sigma}$} & \multirow{14}{*}{ 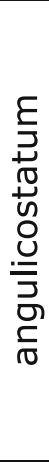 } & AN37b & 1.92 & 21.45 & 0.57 & 2.86 & 5.76 & 0.56 & 42.80 & 100.62 & 50.60 & 12.67 & 26.37 \\
\hline & & AN38b & 1.59 & 14.64 & 0.44 & 3.13 & 5.71 & 0.38 & 38.98 & 81.97 & 38.78 & 13.09 & 16.89 \\
\hline & & AN39b & 1.62 & 16.99 & 1.15 & 4.12 & 6.64 & 1.28 & 36.73 & 167.87 & 42.80 & 14.17 & 16.63 \\
\hline & & AN40b & 2.17 & 16.34 & 0.71 & 3.86 & 6.89 & 0.56 & 44.88 & 139.18 & 42.11 & 22.05 & 16.67 \\
\hline$=$ & & AN41b & 2.56 & 22.10 & 0.73 & 4.47 & 8.67 & 0.84 & 58.35 & 205.46 & 55.25 & 20.63 & 23.53 \\
\hline & & AN42b & 2.28 & 15.30 & 0.16 & 2.99 & 5.63 & 0.58 & 39.87 & 110.99 & 42.70 & 10.33 & 17.90 \\
\hline \multirow{8}{*}{ 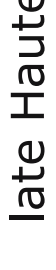 } & & AN43b & 1.59 & 12.39 & 0.27 & 1.73 & 3.39 & 0.35 & 24.57 & 74.26 & 29.27 & 7.90 & 12.99 \\
\hline & & AN44b & 1.58 & 12.78 & 0.28 & 2.41 & 3.88 & 0.34 & 28.36 & 72.99 & 27.64 & 10.51 & 14.37 \\
\hline & & AN45b & 2.32 & 31.12 & 0.57 & 4.80 & 13.38 & 0.32 & 57.41 & 111.72 & 55.01 & 16.73 & 30.66 \\
\hline & & AN46b & 2.15 & 12.78 & 0.25 & 3.45 & 7.80 & 0.39 & 48.73 & 103.01 & 68.49 & 9.22 & 15.47 \\
\hline & & AN48b & 1.85 & 18.98 & 0.37 & 3.35 & 6.26 & 0.49 & 44.40 & 114.71 & 37.86 & 10.54 & 21.80 \\
\hline & & AN51b & 3.54 & 19.23 & 2.97 & 2.06 & 1.82 & 0.06 & 8.39 & 31.71 & 33.60 & 3.61 & 8.42 \\
\hline & & AN52b & 3.73 & 27.35 & 1.78 & 4.71 & 4.19 & 0.45 & 19.08 & 91.12 & 51.41 & 7.13 & 17.08 \\
\hline & & AN53.1b & 2.98 & 18.84 & 3.30 & 1.52 & 1.69 & 0.06 & 10.16 & 19.16 & 38.32 & 3.13 & 7.90 \\
\hline \multirow{3}{*}{$\frac{\grave{L}}{\mathbb{D}}$} & \multirow{3}{*}{$\stackrel{\frac{\pi}{\bar{x}}}{\underline{\underline{x}}}$} & AN72b & 1.59 & 10.45 & 0.37 & 3.20 & 3.72 & 0.22 & 25.72 & 136.80 & 24.11 & 7.30 & 8.84 \\
\hline & & AN73b & 1.61 & 10.84 & 0.31 & 2.94 & 6.00 & 0.50 & 22.91 & 138.40 & 32.25 & 7.44 & 10.76 \\
\hline & & AN75b & 1.48 & 13.92 & 0.19 & 3.39 & 5.66 & 0.29 & 26.22 & 128.48 & 33.94 & 8.73 & 14.48 \\
\hline
\end{tabular}

$\mathrm{V}$ present a double peak in contents around the level of the "couches à poissons," with the higher peak representing the "couches à poissons" itself. The average values of the background levels correspond to approximately $0.5,5.4,0.09$ and $0.8 \mathrm{ppm}$ and the maximum values are approximately $0.9,11.7,1.2$ and $2.5 \mathrm{ppm}$, for $\mathrm{U}, \mathrm{V}, \mathrm{Mo}$ and As contents, respectively. The data obtained in the marls show equally a clear enrichment of U, V, Mo and As around the Faraoni Level (Table 2).

Co contents in limestone show a small overall decrease from the bottom to the top of the section, with a mean value of about $1.7 \mathrm{ppm}$. A peak is distinguished $5 \mathrm{~m}$ below the equivalent of the Faraoni Level, reaching $3.6 \mathrm{ppm}$. In the marls, a peak reaching $7.4 \mathrm{ppm}$ is distinguished $3 \mathrm{~m}$ below the Faraoni Level.

In this section, the $\mathrm{Cu}, \mathrm{Zn}, \mathrm{Ni}$ and $\mathrm{Pb}$ show a small positive shift in the limestone $5 \mathrm{~m}$ below the Faraoni Level equivalent. They correspond to the peaks of $U$, $\mathrm{V}$ and $\mathrm{Co}, \mathrm{Cu}$ and $\mathrm{Zn}$ show equally a peak approximately $40 \mathrm{~m}$ below the Faraoni Level, at the beginning of the B. balearis zone. On the other hand, in the marls, only $\mathrm{Cd}$ is enriched within the Faraoni Level equivalent, whereas enrichment of $\mathrm{Cd}, \mathrm{Zn}, \mathrm{Ni}$ and $\mathrm{Pb}$ characterizes again the beds situated $5 \mathrm{~m}$ below this level. 


\section{The Angles section}

In the limestone samples, the trends in $\mathrm{U}, \mathrm{V}, \mathrm{Mo}$ and As contents are comparable to the other two sections, with peak values at the level of the Faraoni Level equivalent (Fig. 5; Table 1). Whereas the background levels are low and rather constant for U, Mo and As, V contents show a more variable evolution, with a series of positive excursions above the Faraoni Level equivalent. The average values for the background levels are at about 0.7, 4.5, 0.2 and $0.6 \mathrm{ppm}$ and the maximum values correspond to approximately $2.1,8.9,4.9$ and $6.3 \mathrm{ppm}$, for U, V, Mo and As contents, respectively. The data obtained in the marl samples show equally a clear enrichment of U, V, Mo and As around the Faraoni Level equivalent (Table 2).

The overall trend in Co contents in limestone is more or less constant, with average values oscillating around $1.3 \mathrm{ppm}$. A peak with a value of $5.1 \mathrm{ppm}$ is distinguished approximately $6 \mathrm{~m}$ below the equivalent of the Faraoni Level (bed AN 38). In the same bed, a small peak is present in the Mo and the As curves. Within the equivalent of the Faraoni Level, a small peak of Co reaching $2.4 \mathrm{ppm}$ is distinguished. The data obtained in the marls show equally a clear enrichment of U, V, Mo and As around the Faraoni Level equivalent (Table 2). In the marl samples, a remarkable peak in Co contents characterizing the Faraoni Level equivalent has not been observed. A maximum of $13.4 \mathrm{ppm}$ is seen within bed AN 45b.

In this section, a clear peak of $\mathrm{Cd}, \mathrm{Zn}, \mathrm{Ni}$ and $\mathrm{Pb}$ occurs within the limestone beds of the Faraoni Level equivalent. Moreover, a peak of $\mathrm{Ni}$ and $\mathrm{Pb}$ marks bed AN 38. On the other hand, no significant peak of these elements has been found in the Faraoni Level equivalent marls. We notice, however, that all these elements are more concentrated in the marls below the Faraoni equivalent than above.

\section{Total organic carbon}

In the limestone samples, measured TOC values are not higher than $0.1 \%$. We will therefore restrict our discussion to the results obtained in the marl samples (see Fig. 6). In the Fiume-Bosso section, a maximum TOC value of $14.8 \%$ has been measured in the thin marl level interval just below the Faraoni Level. Within the Faraoni Level, the average value of TOC is close to $3.25 \%$. These values are not significantly different from those obtained in marl samples above and below the Faraoni Level.

In the VCD section, no major variations have been measured (Fig. 6). The average value is close to
Table 3 Background level values, maximum value of the peak and enrichment factors of $\mathrm{U}, \mathrm{V}, \mathrm{Mo}$ and As for the three studied sections (see text for calculation details)

\begin{tabular}{llcc}
\hline Ppm & $\begin{array}{l}\text { Background } \\
\text { level average } \\
\text { value }\end{array}$ & $\begin{array}{l}\text { Maximum value } \\
\text { of the peak }\end{array}$ & $\begin{array}{l}\text { Enrichment } \\
\text { factors }\end{array}$ \\
\hline Fiume-Bosso & & \\
U & 0.17 & 3.1 & 18.24 \\
V & 0.9 & 11.6 & 12.89 \\
Mo & 0.03 & 0.55 & 18.33 \\
As & 0.3 & 3 & 10.00 \\
Veveyse & & & \\
U & 0.53 & 0.94 & 1.77 \\
V & 5.411 & 11.7 & 2.16 \\
Mo & 0.09 & 1.22 & 13.56 \\
As & 0.75 & 2.54 & 3.39 \\
Angles & & & \\
U & 0.69 & 2.1 & 3.04 \\
V & 4.55 & 8.9 & 1.96 \\
Mo & 0.24 & 4.87 & 20.29 \\
As & 0.65 & 4.8 & 7.38 \\
\hline
\end{tabular}

$0.5 \%$ and the Faraoni Level equivalent TOC value is equal to $0.31 \%$. In the Angles section, no major variation has been observed as well. The TOC values are however higher and reach $2.63 \%$. TOC values in the Faraoni Level equivalent are close to $1.25 \%$.

\section{Discussion}

Comparison between the three sections

Trends in U, V, Mo and As contents are all characterized by pronounced positive peaks marking the Faraoni Level and its equivalent, and are very distinct relative to the measured intervals below and above this level, representing the upper Hauterivian and the lower Barremian. This positive peak is present in both limestone and marl samples, despite differing background level values. For the trends in $\mathrm{Co}, \mathrm{Cd}, \mathrm{Cu}, \mathrm{Zn}$, $\mathrm{Ni}, \mathrm{Pb}$ and $\mathrm{Cr}$ contents, no significant overall variations are observed and no specific enrichments are recorded for the Faraoni Level and its equivalent apart from local variations.

\section{Behaviour of U, V, Mo, Co and As}

Enrichments in U, V and Mo are well known for numerous black shales of the Phanerozoic (e.g. Brumsack and Gieskes 1983; Brumsack 1989, 2006; Coveney et al. 1991; Sun and Püttmann 1997; Nijenhuis et al. 1999; Warning and Brumsack 2000; Yarincik 
Fig. 3 Redox-sensitive trace elements distributions for the Fiume-Bosso section. The grey band indicates the position of the Faraoni Level and the dashed line the limit of quantification for each element
Fig. 4 Redox-sensitive trace elements distributions for the Veveyse de Châtel-St Denis section. The grey band indicates the position of the "couche à poissons" (Faraoni Level equivalent) and the dashed line the limit of quantification for each element

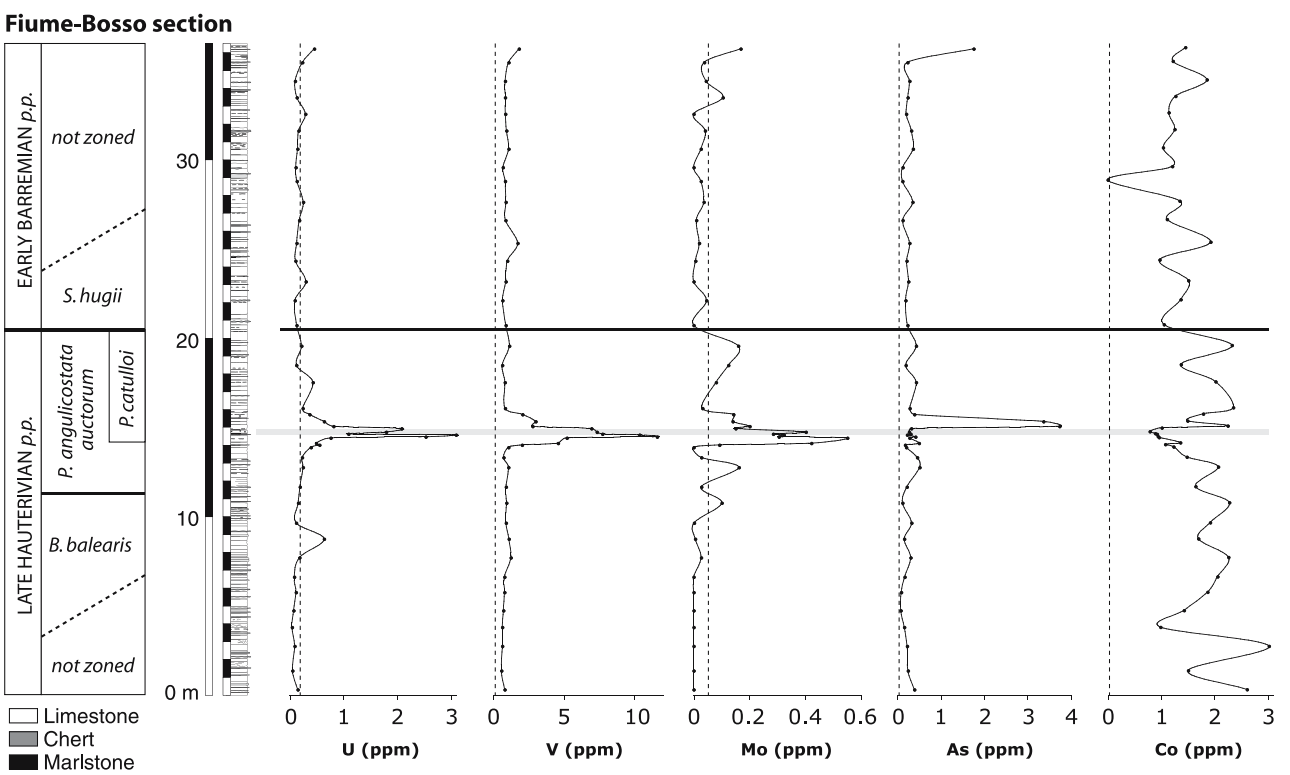

et al. 2000; Mangini et al. 2001; Tribovillard et al. 2004; Wilde et al. 2004). U and V enrichments are usually associated to anoxic to euxinic bottom-water conditions during the time of deposition (e.g. Hastings et al. 1996; Morford and Emerson 1999; Mangini et al. 2001). Under mildly reducing and anoxic conditions, $\mathrm{U}$ and $\mathrm{V}$ may be removed from the ocean by the formation of organo-metal ligands in humic acids; by crystalline precipitation $\left[\mathrm{UO}_{2}\right.$ and $\mathrm{V}_{2} \mathrm{O}_{3}$ or $\left.\mathrm{V}(\mathrm{OH})_{3}\right]$, and $\mathrm{V}$ may also be removed by surface-adsorption processes (Algeo and Maynard 2004). For Mo, Algeo and Maynard (2004) propose adsorption onto humic substances as a main possible mechanism to transfer this element from the water column into the sediment, and under euxinic conditions, by a rapid uptake by authigenic/syngenetic sulfides.

Overall, the solubility of these three elements decreases in the reduced state, and their precipitation and enrichment in sediments is favored during periods of anoxia (e.g. Yarincik et al. 2000; Mangini et al. 2001; Algeo and Maynard 2004).

Co shows a more complex behaviour in the three sections studied here. This element should behave similar to $\mathrm{U}, \mathrm{V}$ and $\mathrm{Mo}$, and indeed, in oxygendepleted waters, Co forms an insoluble sulfide $(\mathrm{CoS})$ that is trapped in sediments (Algeo and Maynard 
Fig. 5 Redox-sensitive trace elements distributions for the Angles section. The grey band indicates the position of the Faraoni Level equivalent and the dashed line the limit of quantification for each element

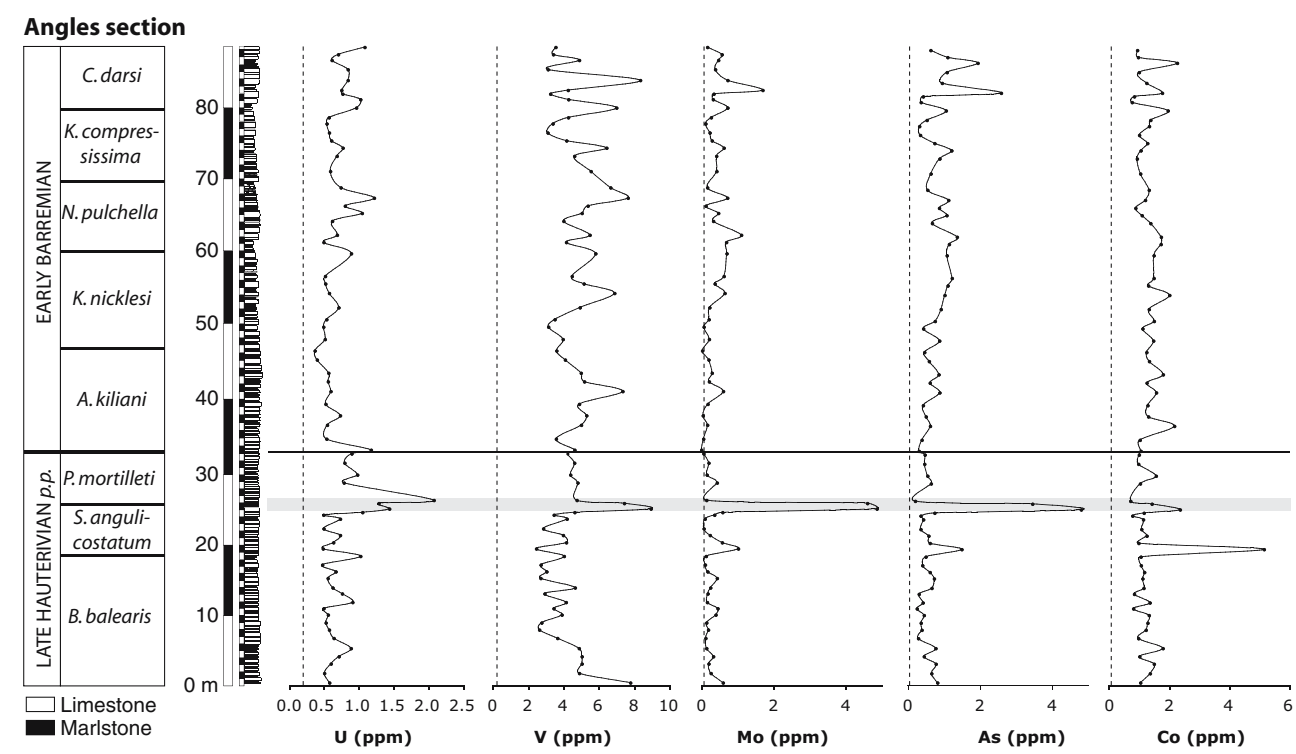

Fig. 6 Correlation of the Faraoni Level for the three different sections. For each section, the total organic carbon analyses in marls as well as the trace element index $\left(\sum E F\right)$ in both limestone and marls are figured. See text for $\sum E F$ calculation details. The lithologic legend for each section is the same as in previous figures
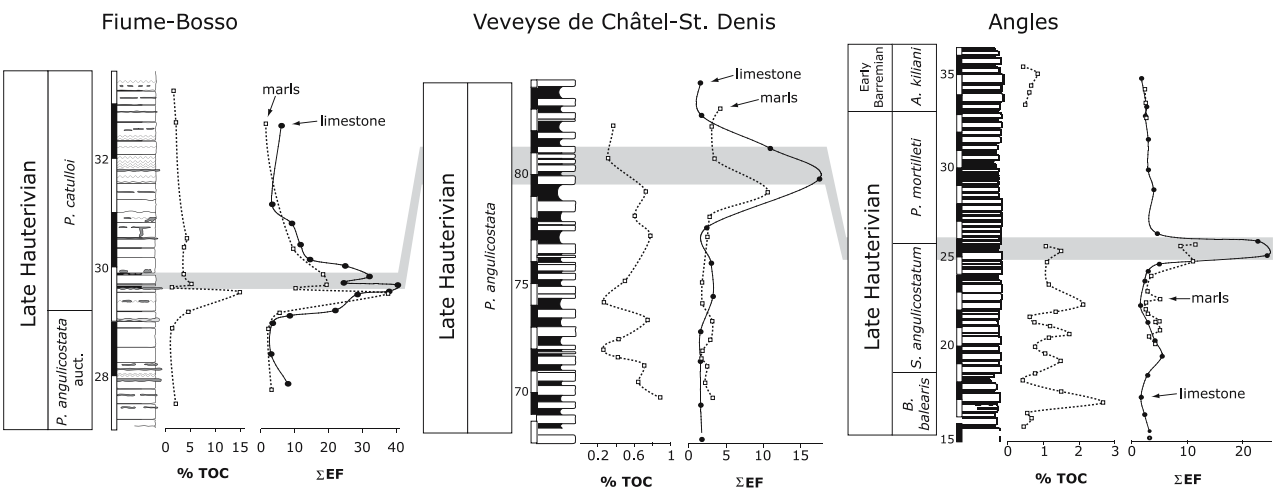

2004). These sulfides are taken up in solid solution by authigenic Fe sulfides (Huerta-Diaz and Morse 1992). But, because of the slow kinetics of this uptake, significant Co enrichment in the sedimentary environment is limited to euxinic conditions (Sun and Püttmann 1997; Algeo and Maynard 2004).

Sedimentary enrichments in As are less well documented from the geological record. As is usually mobile under slightly reducing conditions through the reductive dissolution of the As bearing phases [Fe (hydr)oxides]. Furthermore, Bostick and Fendorf (2003) detailed the mechanisms of As uptake in more anoxic environments and demonstrated the surface precipitation of As on iron sulphide minerals. This hypothesis is supported by Huerta-Diaz and Morse (1992) who noted a close link between As and pyrite in sediments underneath anoxic water. Hence, the presence of abundant marcasite nodules within the Faraoni Level in central Italy (Cecca et al. 1994) is compatible with the enrichment in As.
An increased input of trace metals in the ocean in association with large igneous province (LIP) activity has been forwarded as an alternative explanation for trace-metal enrichment in mid-Cretaceous organic-rich sediments, which were formed during oceanic anoxic events (e.g. Sinton and Duncan 1997; Larson and Erba 1999; Leckie et al. 2002; Erba 2004; Snow et al. 2005). For the late Hauterivian, however, there is no evidence for LIP volcanism so far (e.g. Larson 1991; Courtillot and Renne 2003), although a certain degree of volcanic activity has been documented at Rio Grande, documenting the end of the Paraná continental flood basalts (Stewart et al. 1996). The volume of lava emitted during this episode is one order of magnitude lower compared to other LIPs. Moreover, Brumsack (2006) pointed out that trace-metal enrichment (with the exception of extreme enrichment of $\mathrm{Ag}, \mathrm{Cu}, \mathrm{Ni}, \mathrm{V}$ and $\mathrm{Zn}$ ) in sediments associated with the CenomanianTuronian boundary oceanic anoxic event may be explained by seawater as the mere source for these elements (see also Arthur et al. 1988; Piper 1994; Böning 
et al. 2004). The trace-metal enrichments calculated for the Faraoni event (Table 3) are on the same order of magnitude as those calculated for present-day coastal upwelling zones and anoxic basins (e.g. Brumsack 2006). We therefore exclude volcanic activity as a major source of trace-metal enrichment during the Faraoni event.

\section{Redox conditions during the Faraoni event}

The behaviour of the redox-sensitive elements, measured in and around the Faraoni Level and its equivalents, allows us to precise the redox conditions that were present during the Faraoni event. The enrichment of U, V, Mo and As are interpreted as a signature of anoxic and euxinic conditions (Algeo and Maynard 2004). The conservative behaviour of Co during the Faraoni event with regard to its background level is characteristic of dysoxic to anoxic facies according to these authors. The Faraoni Level is therefore clearly the expression of an anoxic event (with eventually locally weakly euxinic conditions such as is indicated at Angles with regard to a small increase of the Co content). The fact that the other redox-sensitive trace elements studied here are not systematically enriched within the Faraoni Level underlines the limit of our comprehension of this system. Partial diagenetic remobilization or differences in sediment uptake processes may be evoked to explain the non-systematic presence of enrichments in the other studied trace elements. Such remobilization processes are thus thought to be responsible of the slight shift of Mo and As peak within the Angles section. Further investigations are however needed to resolve this problem.

Using total sulphur versus TOC plots, Baudin et al. (2002) argued for dysoxic rather than for truly anoxic conditions for the Faraoni Level. This observation together with the conservative behaviour of Co may hint at the possibility that the anoxic conditions were not so strong and closer to the dysoxia/anoxia threshold that to the anoxia/euxinic threshold $\left(\mathrm{O}_{2}\right.$ close to $0.2 \mathrm{ml} / \mathrm{l}$ of $\mathrm{H}_{2} \mathrm{O}$ ), with the exception of the section at Angles, where conditions may have been more severe.

\section{Local oxygen-deficient conditions below} the Faraoni Level

In the Angles and the VCD sections, isolated maxima in trace-metal contents are recognized, notably below the Faraoni Level equivalent. In the VCD section, U, $\mathrm{V}, \mathrm{Zn}, \mathrm{Ni}$ and Co contents show a peak $5 \mathrm{~m}$ below the Faraoni equivalent. In the Angles section, Mo, As, Ni,
$\mathrm{Pb}$ and Co contents show a peak $6 \mathrm{~m}$ below the Faraoni Level equivalent. These peaks are not correlated in time between the two sections and could have resulted from local changes in bottom-water oxygen conditions, resulting in local anoxia or euxinia.

\section{Enrichment factors}

For each separated lithology (i.e. limestones or marls), enrichment factors (EFs) were calculated for U, V, Mo and As according to the following equation (see Table 3; Fig. 6):

$\mathrm{EFs}=[\mathrm{Me}]_{\text {peak }} /[\mathrm{Me}]_{\text {background }}$

In this equation, $[\mathrm{Me}]_{\text {background }}$ represents the average value of the background level of the different curves and $[\mathrm{Me}]_{\text {peak }}$ is the maximum value of the different peaks. The EFs thus obtained are coherent with those obtained by Morford and Emerson (1999) (for U, V and Mo, and based on references therein) for anoxic conditions, representing another clue for anoxic conditions during the Faraoni event.

In order to compare the different sections, an enrichment factor index ( $\left.\sum \mathrm{EF}\right)$ is calculated by adding the EFs of U, V and Mo (see Fig. 6). A clear peak is thus obtained for the three sections for both lithologies, underlining the higher uptake of $\mathrm{U}, \mathrm{V}$ and $\mathrm{Mo}$ in the sediment during the Faraoni event.

A remarkable feature of the Faraoni Level is the alternation between limestones and laminated shales, which is interpreted, in a first approximation, as the results of variations in oxygen content (see Westphal et al. 2004 for a review). The higher values of redoxsensitive trace elements in the Faraoni shales compared with the Faraoni limestones seem to confirm this hypothesis. However, if we look at the enrichment factors index $\left(\sum E F\right)$ calculated for both marls and limestones (Fig. 6), such a dichotomy is not found. The variation of these indices is more or less the same within the same section. On the contrary, it seems that the limestones are a little bit more enriched in redoxsensitive trace elements than the marls during the Faraoni event compared to "normal" conditions. The higher redox-sensitive trace element content of the marlstones (compared to the limestones) is thus not induced by difference of synsedimentary redox conditions between marlstones and limestones (as already shown by Westphal et al. 2004), but is rather the result of synsedimentary condensation or early diagenetic processes. This underlines the importance of the lithology effect on analyses and the need to consider 
"mono-lithologic" analyses when discussing temporal variations.

In Fig. 6, where the TOC is plotted versus the $\sum E F$, we remark that TOC is only clearly enriched in the Faraoni Level of the Fiume-Bosso section, whereas $\sum E F$ shows a positive shift in all three sections. This allows us to postulate that the enrichment of redoxsensitive trace elements is a more conservative tracer of anoxic conditions than TOC contents. This latter may be more easily removed during diagenetic processes.

\section{Chemostratigraphic tools}

The distinct enrichment of the redox-sensitive elements U, V, Mo and As in the sedimentary environment during the Faraoni event appears to be unique for the examined time interval of the Late Hauterivian-Early Barremian and is therefore useful as a tracer of the Faraoni event within the Tethyan realm.

Outside the Tethyan realm, a distinct enrichment in U contents occurs in sediments of the Late Hauterivian of the Izu-Mariana margin, northwestern Pacific, in ODP hole 1149b (ODP leg 185; Plank et al. 2000, upper part of the lithologic unit IV, interval 185-1149B19R-1; see Figs. F27, F72). This peak is associated with a layer containing elevated quantities of euhedral barite, along with dolomite rhombs and phosphatic fish remains. Plank et al. (2000) argue that this peculiar mineral assemblage indicates an episode of enhanced organic-matter accumulation, which they correlate with the Faraoni event. According to the data presented here, the enrichment in $U$ represents a further strong argument in favor of this assumption. This suggests that the Faraoni event was not restricted to the western Tethyan realm, but may also be recorded in sediments of the northwestern Pacific. Further investigations - especially in sediments of the southern hemisphere-are required to confirm the global character of this event.

In the Fiume-Bosso section, the enrichment in $\mathrm{U}, \mathrm{V}$ and Mo contents began slightly before and ended slightly after the Faraoni Level s.s. This may indicate that the Faraoni anoxic event may have started slightly earlier and lasted longer than the time associated with the formation of the Faraoni Level itself. This would signify that the organic-rich facies of the Faraoni Level in the Italian sections does not reflect the entire period of oxygen depletion, but rather the time of important organic matter preservation and/or accumulation during the culmination of this event. This may be taken into account when tracing the equivalent of the Faraoni Level by using trace-metal enrichment in sections were ammonites are absent or extremely rare. The here described trace-metal enrichments trace the Faraoni anoxic event rather than the Faraoni Level itself.

Initiating factors of the Faraoni event

As recognized by Baudin et al. (2002) and Baudin (2005), the Faraoni Level and its equivalent can be qualified as an anoxic event. An important difference between the Faraoni event and other Cretaceous OAE's consists, however, in the fact that the anoxic event is not mirrored by a major shift in the carbonisotope record (e.g. Erba et al. 1999; Van de Schootbrugge et al. 2000; Company et al. 2005; Godet et al. $2006)$, but only by a small positive excursion $(0.5 \%)$. Godet et al. (2006) explain this attenuated signal by a buffering effect on the carbon-isotope record exerted by the large size of the oceanic dissolved inorganic carbon reservoir. Furthermore, the classical scenario invoking an episode of intensified volcanism and reinforced greenhouse conditions (e.g. Jenkyns 1999, 2003) is difficult to apply to this peculiar event. As previously said, there is no evidence for LIP volcanism during the late Hauterivian so far, even if a certain degree of volcanism has been documented in Rio Grande. This volcanic activity is however one order of magnitude lower than that associated with LIP (e.g. Larson 1991; Courtillot and Renne 2003). We therefore consider the Rio Grande volcanism as not responsible for the Faraoni event, even if slightly stronger greenhouse conditions may have been induced by this volcanic episode.

As discussed by Baudin et al. (2002), the Faraoni Level corresponds to the maximum flooding surface (mfs) of the Ha6 sequence (Haq et al. 1987; Hardenbol et al. 1998). For the VCD section, Busnardo et al. (2003) attributed the "couches à poissons" to the transgressive surface of the Ha6 sequence, but A. Strasser (Fribourg, personal communication) correlates the same horizon also to the $\mathrm{mfs}$ of the $\mathrm{Ha} 6$ sequence (see also Bodin et al. 2006). The Ha6 mfs corresponds to the maximum of a second-order transgression (Haq et al. 1987; Hardenbol et al. 1998), which led to the connection of the Tethyan and the Boreal realms, as is suggested by belemnite migration patterns observed by Mutterlose and Bornemann (2000) and the presence of boreal nannoplankton in the Angles section around the Faraoni Level (Godet et al. 2006). This second-order transgression may have lead to the flooding of large epicontinental areas. Bodin et al. (2006) pointed out the role of this mechanism as the main trigger for the 
onset of anoxic conditions during the Faraoni anoxic event, by increased nutrient delivery, and the related increase in primary productivity (see also Company et al. 2005). Baudin et al. (2002) also proposed increased primary productivity as the prime factor leading to the origin of the Faraoni event along the line of the scenario proposed here.

\section{Conclusions}

Redox-sensitive trace elemental analysis is a fruitful method to better understand and constrain anoxic events. Indeed, the multi-element analysis discussed here provides additional insight into the oxygenation conditions during the Faraoni anoxic event and serves as a promising correlation tool, especially if biostratigraphic information is lacking.

By comparing the behaviour of the redox-sensitive trace elements with the specific enrichments in $\mathrm{U}, \mathrm{V}$ and Mo contents, the Faraoni Level appears to be the expression of anoxic conditions at least near the sediment-water interface. This interpretation is supported by the enrichment of As, which is largely controlled by its sorption onto sulfide minerals in anoxic environments (Bostick and Fendorf 2003). The lack of systematic enrichment of $\mathrm{Cd}, \mathrm{Cu}, \mathrm{Zn}, \mathrm{Ni}, \mathrm{Pb}$ and $\mathrm{Cr}$ may be related to diagenetic removal or unknown synsedimentary differences in uptake processes.

In the sections analysed, the distinct enrichments in $\mathrm{U}, \mathrm{V}$ and Mo are a feature characteristic of the Faraoni Level, and are unique with regards to the time interval of the late Hauterivian and the early Barremian. We therefore propose to use this feature to correlate the Faraoni Level throughout the entire Tethys, and especially in sections where organic-rich intervals are lacking such as in the section of Angles, and to search for expressions of the Faraoni event also in other basins. For example, a distinct enrichment in $U$ in sediments of late Hauterivian age in the northwestern Pacific may well be correlated with the Faraoni event (Plank et al. 2000; see Figs. F27, F72).

According to the general behaviour of the redoxsensitive trace elements in the three analysed sections, the Faraoni Level may be the expression of a climax situation during a longer period of steadily increasing anoxia induced by a second-order sea level rise. The mobilization of nutrients during this sea level rise may have played an important role in the onset of the Faraoni event.
Acknowledgments We thank B. Van de Schootbrugge, H. Mort, P. Linder and C. Rambeau for their help in the field, J. Charollais, H. Mort and F. Baudin for stimulating discussions, T. Monnier for help in the laboratory work and the team of the "Reserve Géologique de Haute-Provence" for providing the authorization for field work and sampling in this region. We also thank E. Erba and J. Hölemann for constructive criticism of the manuscript. Financial support from the Swiss National Science Foundation is gratefully acknowledged (projects 2100-067807/1 and 200020-105206/1).

\section{References}

Algeo TJ, Maynard JB (2004) Trace-element behavior and redox facies in core shales of Upper Pennsylvanian Kansas-type cyclothems. Chem Geol 206:289-318

Arthur MA, Jenkyns HC, Brumsack H-J, Schlanger SO (1988) Stratigraphy, geochemistry, and paleoceanography of organic carbon-rich Cretaceous sequences. In: Ginsburg RN, Beaudoin B (eds) Cretaceous resources, events and rhythms: background and plans for research. Proceedings of ARW, Kluwer Academic Publishers, Digne, France, pp. 75119

Baudin F (2005) A Late Hauterivian short-lived anoxic event in the Mediterranean Tethys: the "Faraoni Event". CR Geosci 337:1532-1540

Baudin F, Bulot LG, Cecca F, Coccioni R, Gardin S, Renard M (1999) Un équivalent du "Niveau Faraoni" dans le bassin du Sud-Est de la France, indice possible d'un événement anoxique fini-hauterivien étendu à la Téthys méditerranéenne. Bull Soc Géol Fr 170:487-498

Baudin F, Cecca F, Galeotti S, Coccioni R (2002) Palaeoenvironmental controls of the distribution of organic matter within a Corg-rich marker bed (Faraoni Level, uppermost Hauterivian, central Italy). Eclogae geologicae Helvetiae 95:1-13

Bayon MM, Alonso JIG, Medel AS (1998) Enhanced semiquantitative multi-analysis of trace elements in environmental samples using inductively coupled plasma mass spectrometry. J Anal At Spectrom 13:277-282

Behar F, Beaumont V, Penteado HLD (2001) Rock-Eval 6 technology: performances and developments. Oil Gas Sci Technol 56:111-134

Bodin S, Godet A, Föllmi KB, Vermeulen J, Arnaud H, Strasser A, Fiet N, Adatte T (2006) The Late Hauterivian Faraoni oceanic anoxic event in the western Tethys: evidence from phosphorus burial rates. Palaeogeogr Palaeoclimatol Palaeoecol 235:238-257

Böning P, Brumsack H-J, Bottcher ME, Schnetger B, Kriete C, Kallmeyer J, Borchers SL (2004) Geochemistry of Peruvian near-surface sediments. Geochim Cosmochim Acta 68:4429-4451

Bostick BC, Fendorf S (2003) Arsenite sorption on troilite (FeS) and pyrite (FeS2). Geochim Cosmochim Acta 67:909-921

Brumsack H-J (1989) Geochemistry of recent TOC-rich sediments from the Gulf of California and the Black Sea. Geol Rundsch 78:851-882

Brumsack H-J (2006) The trace metal content of recent organic carbon-rich sediments: implications for Cretaceous black shale formation. Palaeogeogr Palaeoclimatol Palaeoecol 232:344-361

Brumsack H-J, Gieskes JM (1983) Interstitial water trace-element chemistry of laminated sediments of the Gulf of California (Mexico). Mar Chem 14:89-106 
Busnardo R (1965) Le stratotype du Barrémien. Mém Bur Rech Géol Min 34:101-116

Busnardo R, Charollais J, Weidmann M, Clavel B (2003) Le Crétacé inférieur de la Veveyse de Châtel (Ultrahelvétique des Préalpes externes; canton de Fribourg, Suisse). Rev Paléobiol Genève 22:1-174

Cecca F, Marini A, Pallini G, Baudin F, Begouen V (1994) A guide-level of the uppermost Hauterivian (Lower Cretaceous) in the pelagic succession of Umbria-Marche Apennines (Central Italy): the Faraoni Level. Riv Ital Paleontol Stratigr 99:551-568

Cecca F, Galeotti S, Coccioni R, Erba E (1996) The equivalent of the "Faraoni Level" (uppermost Hauterivian, Lower Cretaceous) recorded in the eastern part of Trento Plateau (Venetian southern Alps, Italy). Riv Ital Paleontol Stratigr 102:417-424

Coccioni R, Baudin F, Cecca F, Chiari M, Galeotti S, Gardin S, Salvini G (1998) Integrated stratigraphic, palaeontological, and geochemical analysis of the uppermost Hauterivian Faraoni Level in the Fiume Bosso section, Umbria-Marche Apennines, Italy. Cretaceous Res 19:1-23

Company M, Aguado R, Sandoval J, Tavera JM, Jiménez de Cisneros C, Vera JA (2005) Biotic changes linked to a minor anoxic event (Faraoni Level, latest Hauterivian, Early Cretaceous). Palaeogeogr Palaeoclimatol Palaeoecol 224:186-199

Courtillot VE, Renne PR (2003) On the ages of flood basalt events. C R Geosci 335:113-140

Coveney JRM, Lynn Watney W, Maples CG (1991) Contrasting depositional models for Pennsylvanian black shale discerned from molybdenum abundances. Geology 19:147-150

Erba E (2004) Calcareous nannofossils and Mesozoic oceanic anoxic events. Mar Micropaleontol 52:85-106

Erba E, Channell JET, Claps M, Jones CE, Larson RL, Opdyke B, Premoli-Silva I, Riva A, Salvini G, Torriceli S (1999) Integrated stratigraphy of the Cismon APTICORE (Southern Alps, Italy): a "reference section" for the Barremian-Aptian interval at low latitudes. J Foraminiferal Res 29:371-391

Erba E, Bartolini A, Larson RL (2004) Valanginian Weissert oceanic anoxic event. Geology 32:149-152

Föllmi KB, Weissert H, Bisping M, Funk H (1994) Phosphogenesis, carbon-isotope stratigraphy, and carbonate-platform evolution along the Lower Cretaceous northern Tethyan margin. Geol Soc Am Bull 106:729-746

Godet A, Bodin S, Föllmi KB, Vermeulen J, Gardin S, Fiet N, Adatte T, Berner Z, Stüben D, Van de Schootbrugge B (2006) Evolution of the marine stable carbon-isotope record during the early Cretaceous: a focus on the late Hauterivian and Barremian in the Tethyan realm. Earth Planet Sci Lett 242:254-271

Haq BU, Hardenbol J, Vail PR (1987) Chronology of fluctuating sea levels since the Triassic. Science 235:1156-1167

Hardenbol J, Thierry J, Farley MB, de Graciansky P-C, Vail PR (1998) Mesozoic and cenozoic sequence chronostratigraphic framework of European Basins. In: de Graciansky P-C, Hardenbol J, Jacquin T, Vail PR (eds) Mesozoic and cenozoic sequence stratigraphy of European Basins. Special Publication Society for Sedimentary Geology, pp. 313

Hastings DW, Emerson SR, Mix AC (1996) Vanadium in foraminiferal calcite as a tracer for changes in the areal extend of reducing sediments. Paleoceanography 11:665-678

Hay WW, DeConto R, Wold CN, Wilson KM, Voigt S, Schulz M, Wold-Rossby A, Dullo W-C, Ronov AB, Balukhovsky AN, Soeding E (1999) Alternative global Cretaceous paleogeography. In: Barrera E, Johnson C (eds) The evolution of Cretaceous ocean/climate systems. Geological Society of America Special Paper, p. 332

Huerta-Diaz MA, Morse JW (1992) Pyritization of trace metals in anoxic marine sediments. Geochim Cosmochim Acta $56: 2681-2702$

Jenkyns HC (1999) Mesozoic anoxic events and palaeoclimate. Zentralbl Geol Paläontol 1 Heft 7-9:943-949

Jenkyns HC (2003) Evidence for rapid climate change in the Mesozoic-Palaeogene greenhouse world. Philos Trans R Soc Lond A 361:1885-1916

Jitaru P, Tirez K, De Brucker N (2003) Panoramic analysis for monitoring trace metals in natural waters by ICP-MS. At Spectr 24:1-10

Jones CE, Jenkyns HC (2001) Seawater strontium isotopes, oceanic anoxic events, and seafloor hydrothermal activity in the Jurassic and Cretaceous. Am J Sci 301:112-149

Larson RL (1991) Latest pulse of Earth: evidence for a midCretaceous superplume. Geology 19:547-550

Larson RL, Erba E (1999) Onset of the mid-Cretaceous greenhouse in the Barremian-Aptian: igneous events and the biological, sedimentary and geochemical responses. Paleoceanography 14:663-678

Leckie RM, Bralower TJ, Cashman R (2002) Oceanic anoxic events and plankton evolution: biotic response to tectonic forcing during the mid-Cretaceous. Paleoceanography 17:PA000623

Lini A, Weissert H, Erba E (1992) The Valanginian carbon isotope event: a first episode of greenhouse climate conditions during the Cretaceous. Terra Nova 4:374-384

Mangini A, Jung M, Laukenmann S (2001) What do we learn from peaks of uranium and of manganese in deep sea sediments? Mar Geol 177:63-78

Morford JL, Emerson SR (1999) The geochemistry of redox sensitive trace metals in sediments. Geochim Cosmochim Acta 63:1735-1750

Mutterlose J, Bornemann A (2000) Distribution and facies patterns of Lower Cretaceous sediments in northern Germany: a review. Cretaceous Res 21:733-759

Nijenhuis IA, Bosch H-J, Sinninghe Damste JS, Brumsack H-J, De Lange GJ (1999) Organic matter and trace element rich sapropels and black shales: a geochemical comparison. Earth Planet Sci Lett 169:277-290

Piper DZ (1994) Seawater as the source of minor elements in black shales, phosphorites and other sedimentary rocks. Chem Geol 114:95-114

Plank T, Ludden JN, Escutia C et al (2000) Proceeding of the Ocean Drilling Program, Initial Reports, 185 [Online], Available from World Wide Web: http://www-odp.tamu.edu/publications/185_IR/185ir.htm

Sanfourche J, Baudin F (2001) La genèse des évènements anoxiques de la période moyenne du Crétacé. Examen de l'hypothèse du meromictisme océanique. Ann Soc Géol Nord 8:107-119

Schlanger SO, Jenkyns HC (1976) Cretaceous oceanic anoxic events: causes and consequences. Geologie en Mijnbouw 55:179-184

Schlanger SO, Arthur MA, Jenkyns HC, Scholle PA (1987) The Cenomanian-Turonian oceanic anoxic event. I. Stratigraphy and distribution of organic-rich beds and the marine ${ }^{13} \mathrm{C}$ excursion. In: Brooks J, Fleet AJ (eds) Marine petroleum sources rocks. Geological Society, London, Special Publications, pp. 371-399

Sinton CW, Duncan RA (1997) Potential links between ocean plateau volcanism and global ocean anoxia at the Cenomanian-Turonian boundary. Econ Geol 92:836-842 
Snow LJ, Duncan RA, Bralower TJ (2005) Trace element abundances in the Rock Canyon Anticline, Pueblo, Colorado, marine sedimentary section and their relationship to Caribbean plateau construction and oxygen anoxic event 2. Paleoceanography 20:PA3005

Stewart K, Turner S, Kelley S, Hawkesworth C, Kirstein L, Mantovani M (1996) 3-D, ${ }^{40} \mathrm{Ar}-{ }^{39} \mathrm{Ar}$ geochronology in the Paraná continental flood basalt province. Earth Planet Sci Lett 143:95-109

Sun Y, Püttmann W (1997) Metal accumulation during and after deposition of the Kupferschiefer from the Sangerhausen Basin, Germany. Appl Geochem 12:577-592

Tribovillard N, Riboulleau A, Lyons T, Baudin F (2004) Enhanced trapping of molybdenum by sulfurized marine organic matter of marine origin in Mesozoic limestones and shales. Chem Geol 213:385-401

Trümpy R (1960) Paleotectonic evolution of the Central and Western Alps. Bull Geol Soc Am 71:843-908

Van de Schootbrugge B, Föllmi KB, Bulot LG, Burns SJ (2000) Paleoceanographic changes during the early Cretaceous (Valanginian-Hauterivian): evidence from oxygen and carbon stable isotopes. Earth Planet Sci Lett 181:15-31
Van de Schootbrugge B, Kuhn O, Adatte T, Steinmann P, Föllmi KB (2003) Decoupling of P- and Corg-burial following Early Cretaceous (Valanginian-Hauterivian) platform drowning along the NW Tethyan margin. Palaeogeogr Palaeoclimatol Palaeoecol 199:315-331

Warning B, Brumsack H-J (2000) Trace metal signatures of eastern Mediterranean sapropels. Palaeogeogr Palaeoclimatol Palaeoecol 158:293-309

Westphal H, Munnecke A, Pross J, Herrle JO (2004) Multiproxy approach to understanding the origin of Cretaceous pelagic limestone-marl alternations (DSDP site 391, Blake-Bahama Basin). Sedimentology 51:109-126

Wilde P, Lyons TW, Quinby-Hunt MS (2004) Organic carbon proxies in black shales: molybdenum. Chem Geol 206:167176

Yarincik KM, Murray RW, Lyons TW, Peterson LC, Haug GH (2000) Oxygenation history of bottom waters in the Cariaco Basin, Venezuela, over the past 578,000 years: results from redox-sensitive metals ( $\mathrm{Mo}, \mathrm{V}, \mathrm{Mn}$, and $\mathrm{Fe}$ ). Paleoceanography 15:593-604 